
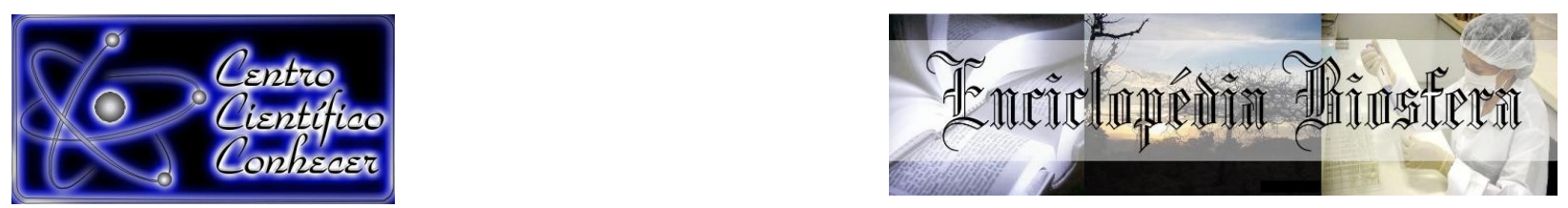

\title{
SUSTENTABILIDADE LABORATORIAL: UMA INICIATIVA DE REUTILIZAÇÃO DE PRODUTOS QUÍMICOS VENCIDOS
}

Ana Carolina de Oliveira Soares ${ }^{1}$; Francisca Lívia de Oliveira Machado ${ }^{2}$; Juliana Monteiro da Silva ${ }^{3}$; Suzana Claudia Silveira Martins ${ }^{4}$; Claudia Miranda Martins ${ }^{5}$

${ }^{1}$ Bióloga graduada pela Universidade Federal do Ceará

${ }^{2}$ Tecnóloga em Química na Universidade Federal do Ceará

${ }^{3}$ Técnica de laboratório(Química) na Universidade Federal do Ceará

${ }^{4}$ Prof ${ }^{a}$ Doutora do Departamento de Biologia da Universidade Federal do Ceará

Fortaleza-Brasil

${ }^{5}$ Prof ${ }^{a}$ Doutora do Departamento de Biologia da Universidade Federal do Ceará

(claudia.miranda.martins@gmail.com) Fortaleza-Brasil

Recebido em: 06/04/2018 - Aprovado em: 10/06/2018 - Publicado em: 20/06/2018 DOI: 10.18677/EnciBio_2018A95

\begin{abstract}
RESUMO
O presente trabalho está baseado numa perspectiva de sustentabilidade ambiental, por meio do gerenciamento e reutilização de alguns produtos químicos anteriormente considerados rejeitos, por apresentarem data de validade expirada, porém com aspecto visual ainda dentro das características originais (rótulo, recipiente e tampa em condições apropriadas; conservados em ambiente sob refrigeração). Esses produtos estavam presentes no Laboratório de Microbiologia Ambiental (LAMAB) do Departamento de Biologia da Universidade Federal do Ceará (UFC), laboratório este utilizado anteriormente para fins didáticos. Com a mudança, foi observado grande volume de passivo ambiental sem nenhum critério de gerenciamento, dentre os quais se encontravam resíduos com as seguintes características: sem identificação; reagentes químicos rotulados, com propriedades físicas conservadas e prazos de validade expirados; reagentes químicos rotulados, com propriedades físicas não conservadas (produtos originalmente líquidos, apresentando sedimentos; produtos sólidos apresentando umidade excessiva ou totalmente liquefeitos). Com o apoio do Programa de Gerenciamento de Resíduos (PROGERE), programa de extensão da Universidade Federal do Ceará, foi adotado um modelo de gerenciamento para tais resíduos de modo a possibilitar a reutilização de parte deles bem como propiciar maior segurança aos usuários do laboratório, tendo em vista que a separação desses produtos foi realizada, baseando-se no grau de incompatibilidade química, existente entre esses produtos, contribuindo assim para minimizar o impacto no meio ambiente e garantir maior segurança aos usuários do LAMAB.
\end{abstract}

PALAVRAS-CHAVE: gerenciamento, reaproveitamento, resíduos 


\title{
LABORATORY SUSTAINABILITY: AN INITIATIVE OF REUSE OF EXISTING CHEMICALS
}

\begin{abstract}
The present work is based on an environmental sustainability perspective, through the management and reuse of some chemical products previously considered waste, due to expired date of expiration, but with a visual aspect still within the original characteristics (label, container and cover under appropriate conditions stored refrigerated). These chemicals were present at the Laboratório de Microbiologia Ambiental (LAMAB) of the Department of Biology of the Universidade Federal do Ceará (UFC), a laboratory used previously for didactic purposes. With the change, a large volume of environmental liabilities was observed without any management criteria, among which were residues with the following characteristics: without identification; labeled chemical reagents with preserved physical properties and expired expiration dates; labeled chemical reagents with uncorrected physical properties (originally liquid products with sediments, solid products with excessive or totally liquefied moisture). With the support of the Programa de Gerenciamento de Resíduos (PROGERE), an extension program of the UFC, a management model was adopted for these residues, in order to allow the reuse of part of them as well as to provide greater safety for laboratory users, considering that the separation and reorganization of these products was made on the basis of the chemical incompatibility existing between these products, thus helping to minimize the impact on the environment and to guarantee greater safety to LAMAB users.
\end{abstract}

KEY WORDS: management, reuse, waste

\section{INTRODUÇÃO}

No Brasil, ocorreu aumento significativo de instituições de ensino superior desde meados de 1990 (BARROS, 2015). Isso implica em provável aumento na geração de resíduos provenientes das atividades de ensino, pesquisa e extensão, especialmente resíduos químicos tais como rejeitos de aulas experimentais e de pesquisas. Dentre as atividades desenvolvidas nessas instituições, estão as aulas experimentais, onde comumente são gerados resíduos químicos, biológicos e radioativos, com alto grau de contaminação e toxicidade. Ocorre que, na maioria das vezes, não existe plano de gestão para esses resíduos, os quais acabam sendo descartados de modo incorreto no meio ambiente (MACHADO; MÓL, 2008).

Dentre os diversos resíduos gerados, existem os que são considerados perigosos (ASSOCIAÇÃO BRASILEIRA DE NORMAS TÉCNICAS, 2004) e podem acarretar riscos de acidentes ambientais, com ou sem a contribuição humana, e diante disso, tem sido crescente o desenvolvimento de metodologias sustentáveis de manejo dos resíduos, que são baseadas no conceito da Química Verde (FERREIRA et al., 2016).

Além da importância na adoção de metodologias sustentáveis, ocorrem discussões coletivas, projetos de pesquisas, estratégias de ações, estabelecimento de protocolos de minimização de resíduos, dentre outras, cada vez mais aplicadas no sentido de solucionar os problemas relacionados ao descarte indevido de resíduos, na maioria das vezes tóxicos, buscando soluções como redução da geração e reutilização, ao invés do descarte incorreto (NASCIMENTO; FILHO, 2010; 
KER et al., 2017). Há quase duas décadas, muitos trabalhos vêm sendo realizados no sentido de buscar medidas e soluções, além de propor procedimentos de gestão dos resíduos (JARDIM, 1998; NOLASCO et al., 2006).

O poder público instituiu a Política Nacional dos Resíduos Sólidos, Lei $N^{\circ}$ 12.305/2010, que enfatiza, dos artigos 25 ao 29, as responsabilidades dos geradores de resíduos, sendo um instrumento importante na busca do comprometimento dos mais diversos atores da sociedade em buscar soluções que sejam sustentáveis na gestão de seus resíduos (BRASIL, 2010).

A Universidade Federal do Ceará como ente inserida nesse contexto, tem realizado pesquisas, embora de forma isolada, com alguns projetos já presentes em alguns dos diversos setores (SILVA et al., 2015). Tendo em vista as especificidades dos resíduos gerados, de acordo com o tipo de laboratório, é importante que cada laboratório individualmente tenha mecanismos de gerenciamento que atendam às suas particularidades. Assim, o presente trabalho está baseado na gestão posterior de produtos químicos, estocados inadequadamente no Laboratório de Microbiologia Ambiental, antigo Laboratório Didático de Microbiologia, sendo esses produtos compostos por: reagentes com prazo de validade vencido, porém aparentemente em condições de uso, visto que o estado físico desses reagentes estavam de acordo com as originais, além dos rótulos e recipientes estarem em boas condições; produtos químicos considerados rejeitos por não apresentarem rótulos de identificação; produtos químicos vencidos, com rótulos intactos, porém apresentando características diferentes das originais, os quais também foram considerados rejeitos.

A segregação de produtos químicos, com critérios de segurança, é realizada de acordo com a incompatibilidade química, baseando-se na ficha de informações de segurança de produtos químicos (FISPQ) de cada produto. Essas fichas fornecem informações sobre medidas de proteção, segurança, saúde, meio ambiente, perigos associados ao produto e as suas incompatibilidades químicas, tornando possível a segregação de forma segura de produtos e resíduos dessa natureza (ASSOCIAÇÃO BRASILEIRA DE NORMAS TÉCNICAS, 2009).

Com base nisso, o principal objetivo da pesquisa foi, além de promover a segregação dos produtos por incompatibilidade química, disponibilizar para a comunidade acadêmica os reagentes passíveis de reutilização, por apresentarem boas condições em seus aspectos físicos, para fins menos nobres. Desse modo, esses produtos já considerados resíduos, puderam ser reutilizados ao invés de serem descartados no meio ambiente.

\section{MATERIAL E MÉTODOS}

Esse trabalho foi realizado no período de julho de 2015 a janeiro de 2016 no Laboratório de Microbiologia Ambiental (LAMAB) do Departamento de Biologia da Universidade Federal do Ceará, com o apoio do Programa de Gerenciamento de Resíduos (PROGERE), programa de extensão da Universidade Federal do Ceará por meio do seguinte processo:

\section{- Inventário}

Primeiramente, foi realizado um inventário de todos os materiais passivos do laboratório, sendo esses produtos catalogados com as seguintes informações:

2.1.1 nome ou formula química;

2.1.2 quantidade;

2.1.3 forma de acondicionamento (vidro, plástico ou outro); 
2.1.4 outras informações, como estado de conservação do recipiente, estado físico do material, condições do rótulo, lote e data de validade.

- Classificação

Os materiais passivos inventariados foram classificados como Reagentes Identificados, Resíduos e Resíduos não identificados (JARDIM, 1998), utilizando-se os seguintes critérios:

1. Reagentes Identificados: material passivo com rótulos originais do fabricante, ou com informações que tornaram possível essa identificação e em condições de uso, ou seja, com suas características físico-químicas conservadas.

2. Resíduos: material passivo identificado, porém sem condições de uso; soluções; meios de culturas usados em microbiologia; ou recipientes identificados que estavam abertos.

3. Resíduos Não Identificados: material passivo sem qualquer identificação.

\section{- Rotulagem}

Para os Reagentes Identificados e Resíduos que se encontravam sem rótulo ou com o rótulo desgastado (amarelado ou caindo), foi feita uma nova rotulagem dos mesmos. Os Resíduos Não Identificados foram rotulados como tal. As informações constantes no rotulo são: nome do reagente, fórmula química e quantidade.

\section{- Armazenamento}

A incompatibilidade química leva em consideração a segregação de produtos químicos conforme suas propriedades físicas e químicas. Desse modo, o material foi separado e acondicionado em diversas caixas mediante sua incompatibilidade química.

As informações para tanto foram obtidas nas em tabelas de incompatibilidade química fornecidas pelo Programa de Gerenciamento de Resíduos da Universidade Federal do Ceará (PROGERE) e Fichas de Segurança de Produtos Químicos (FISPQ).

\section{- Tratamento e destinação final ambientalmente adequada}

Os Reagentes Identificados foram adicionados ao Banco de Reagentes e Utensílios Laboratoriais do Programa de Gerenciamento de Resíduos/Divisão de Gestão Ambiental da UFC, que tem como objetivo o remanejamento de reagentes e utensílios excedentes de alguns laboratórios da UFC para outros laboratórios que precisam desses materiais.

Com relação aos Resíduos Identificados e Não Identificados foi realizado levantamento bibliográfico através das FISPQ sobre os possíveis tratamentos e destinação final ambientalmente adequada os mesmos.

\section{RESULTADOS E DISCUSSÃO}

No inventário do material passivo do $L A M A B$ foram contabilizados 235 frascos, sendo 173 Reagentes e 62 Resíduos. Em relação ao acondicionamento, 
foram observados materiais: acondicionados em frascos inadequados (Figura 1A), como por exemplo, em frascos de reaproveitado de produtos alimentícios; com rótulos avariados (Figura 1B); sem identificação (Figura 1C); com embalagens comprometidas ou incompletas, como por exemplo, frascos sem tampa (Figura 1D). Além de materiais com o estado físico alterado, de acordo com o descrito no rótulo. $\mathrm{Na}$ Figura 1E podemos temos uma visão geral de alguns dos materiais encontrados que estavam acondicionados de maneira inadequada.

\begin{tabular}{|l|l|}
\hline $\begin{array}{l}\text { (A) Material acondicionado em frasco } \\
\text { inadequado. }\end{array}$ & (B) Material com o rótulo avariado \\
\hline &
\end{tabular}

Figura 1: Produtos químicos acondicionados de maneira inadequada.

Os frascos que apresentavam rótulos avariados ou sem rótulo receberam nova identificação (Figura 2). 


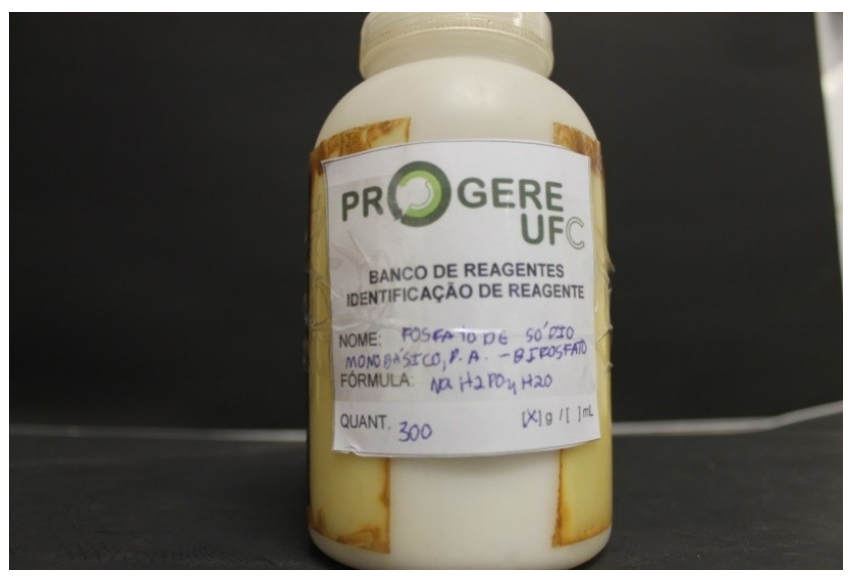

Figura 2: Produtos químicos com nova identificação.

Os reagentes foram segregados em quinze caixas de acordo com a compatibilidade química de cada um. Andrade et al. (2015) destacaram em seu trabalho Segregação de Resíduos Químicos por Compatibilidade e Reatividade, no Instituto Butantan, que a segregação correta dos resíduos químicos está ligada diretamente à a destinação final segura e que os resíduos químicos, assim como os produto devem ser segregados de acordo com suas propriedades físico-químicas e reatividade, evitando que as substâncias incompatíveis entre si sejam armazenadas e transportadas juntas e acidentalmente entrem em contato, podendo reagir violentamente e causar uma explosão ou gerar gases tóxicos ou inflamáveis.

Desde modo os reagentes foram segregados em 15 caixas de acordo com a compatibilidade química de cada um, para isso foi foram utilizadas tabelas de compatibilidade química e FISPQ dos produtos em questão.

Com isso, a segregação dos materiais desse trabalho foi organizada nas caixas de acordo com o Quadro 1.

Quadro 1: Segregação de reagentes segundo a compatibilidade química.

\begin{tabular}{|c|c|}
\hline Caixa & Conteúdo \\
\hline 1 & $\begin{array}{l}\text { ácidos orgânicos, sulfatos, indicadores, cloretos, iodetos, corantes e sais } \\
\text { orgânicos }\end{array}$ \\
\hline 2 & corantes, tolueno e xilol \\
\hline 3 & iodo \\
\hline 4 & sulfatos e carbonatos, molibdatos e corantes \\
\hline 5 & $\begin{array}{l}\text { sulfatos, tiossulfatos, hidróxidos, corantes, oxalatos, orgânicos não } \\
\text { clorados, sulfitos, dicromatos, sais orgânicos }\end{array}$ \\
\hline 6 & Fosfatos \\
\hline 7 & cloretos, ácidos orgânicos e inorgânicos e sulfatos \\
\hline 8 & $\begin{array}{l}\text { citratos, ácidos orgânicos e inorgânicos, orgânicos não halogenados, } \\
\text { nitrito, cloretos, sais de telúrio e tungstênio e meio de cultura para } \\
\text { microbiologia e aminoácido }\end{array}$ \\
\hline 9 & ácidos orgânicos, bromato, tetraborato e fosfato \\
\hline 10 & álcoois, óleo e orgânicos não clorados \\
\hline 11 & reagente de Kovac's \\
\hline 12 & etilbenzeno \\
\hline 13 & 1-naftol (alfa) \\
\hline 14 & tetracloreto de carbono \\
\hline
\end{tabular}


Os Reagentes Identificados foram incluídos no Banco de Reagentes e Utensílios Laboratoriais (BRUL) do PROGERE-UFC para a reutilização em outros laboratórios. Segundo Silva et al. (2015), a logística do BRUL é feita em algumas etapas. Primeiramente o laboratório que tem a intenção de doar os seus reagentes deve preencher o Formulário de Solicitação de Inventário, o qual está disponível no site do PROGERE (www.progere.ufc.br). Após isso, o laboratório deve aguardar o retorno com agendamento para o inventário e a avaliação dos reagentes.

Durante esse processo, os reagentes que ainda apresentam as características originais, ou seja, que estão adequados para o uso, são catalogados e ficam armazenados no ponto gerador até que sejam doados. Segundo o laboratório interessado em receber os reagentes deve acessar o site do PROGERE e verificar no catálogo on-line Banco de Reagentes e Utensílios Laboratoriais (lista com reagentes para doação) se há disponibilidade dos materiais desejados e, em seguida, preencher o Formulário de Solicitação de Reagentes. Então, os reagentes são remanejados para o laboratório solicitante. De acordo com http://www.progere.ufc.br/gerenciamento-de-residuos/

https://drive.google.com/file/d/0Bx0w6MPYkYu7cEFhT2J4S1N4Ync/view responsável pelo laboratório solicitante assina um termo de compromisso aonde mostra está ciente de: 1) os materiais recebidos podem estar fora do prazo de validade, não apresentarem todas as propriedades físico-químicas descritas no rótulo, ou estarem em quantidade inferior ao descrito no rótulo; 2) os materiais recebidos devem ser manuseados apenas por pessoal capacitado; 3) solicitar somente a quantidade de materiais realmente necessários e de destinar adequadamente qualquer resíduo oriundo do(s) produtos(s) doado(s).

Dos 173 reagentes cadastrados, os quais $38693 \mathrm{~g}$ e $21345 \mathrm{~mL}$ foram doados 105 reagentes, onde $29288 \mathrm{~g}$ e $8995 \mathrm{~mL}$, perfazendo 60,69\% dos Reagentes Identificados. A Figura 3 apresenta os resultados com relação aos reagentes sólidos e a Figura 4 com relação aos reagentes líquidos. As doações ocorreram no período de 20 de outubro de 2016 até 19 de abril de 2017.

Reagentes sólidos doados $\mathrm{x}$ total cadastrados

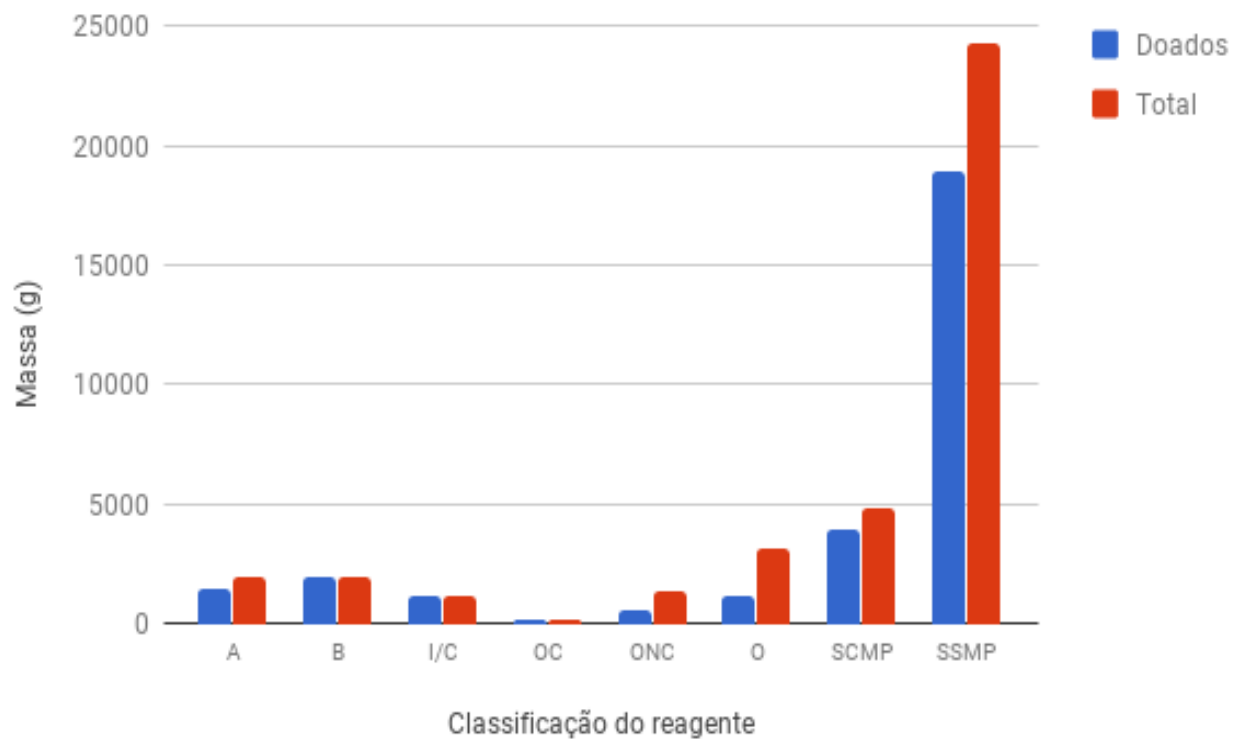


Figura 3: Reagentes sólidos doados versus total cadastrados em massa(g). Legenda: A: ácido; B: base; I/C: indicador/corante; OC: orgânico clorado; ONC: orgânico não clorado; O: outros; SCMP: sal com metal pesado; SSMP: sal sem metal pesado.

\section{Reagentes líquidos doados $\mathbf{x}$ total cadastrados}

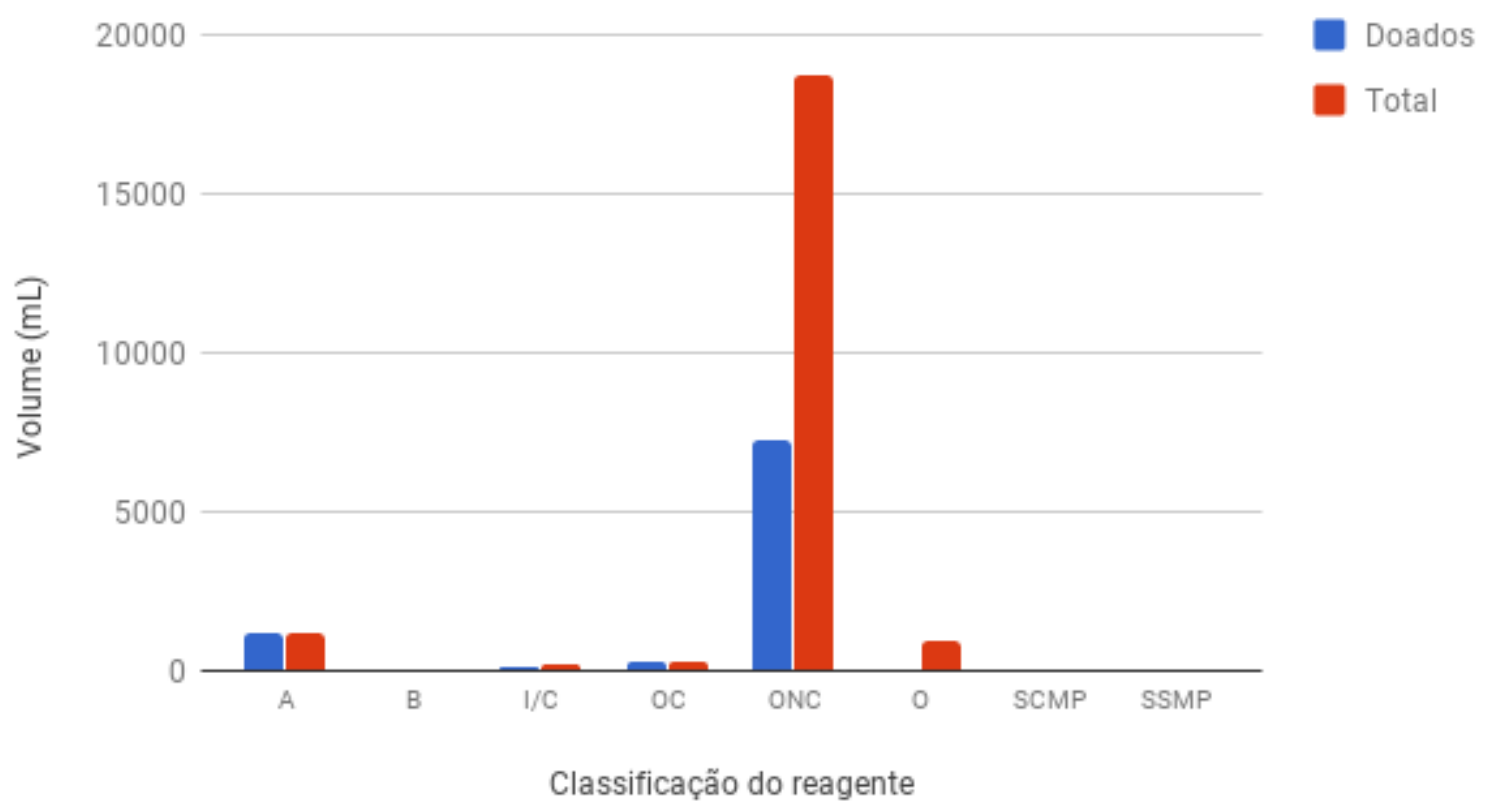

Figura 4: Reagentes líquidos doados versus total cadastrados em massa(g). Legenda: A: ácido; B: base; I/C: indicador/corante; OC: orgânico clorado; ONC: orgânico não clorado; O: outros; SCMP: sal com metal pesado; SSMP: sal sem metal pesado.

As doações ocorreram no período de 20 de outubro de 2016 até 19 de abril de 2017. Cerca de $50 \%$ dos reagentes foram doados em apenas seis meses, o que representa um curto período de tempo. Pode-se verificar que os reagentes que tiveram maior rotatividade foram os sais sem metal pesado, que são reagentes em sua maioria simples e não perigosos utilizados em diversas áreas da ciência. Com relação aos reagentes classificados como Outros não apresentaram uma boa taxa de doação, isso pode ser devido a especificidade desses reagentes, pois estes, em sua maioria, são reagentes exclusivos para pesquisa na área da biologia, como por exemplo o reagente de Kovac's, utilizado para a detecção indol em testes bioquímicos utilizados na identificação de micro-organismos, diminuindo assim, as chances de doações desses materiais.

Os Reagentes Identificados foram doados para oito laboratórios em diversas cidades do Estado do Ceará, dos quais:

- Fortaleza:

Universidade Federal do Ceará-Campus do Pici: Departamento de Química Orgânica e Inorgânica (1 laboratório), Departamento de Silvicultura/Fitotecnia (1 laboratório) e Departamento de Engenharia de Alimentos (1 laboratório), Campus Crateús (1 laboratório).

- Acaraú: Instituto Federal do Ceará-Campus Acaraú: Curso de Engenharia (1 laboratório);

- Redenção: 
Universidade da Integração Internacional da Lusofonia Afro-Brasileira: Instituto de Ciência da Natureza e Matemática (2 laboratórios);

- Pacatuba:

Escola de Ensino Fundamental e Médio Desembargador Raimundo de Carvalho Lima.

Por meio deste projeto os materiais passivos do laboratório, ou seja, materiais armazenados no laboratório sem uso algum, agora estão sendo úteis ao ensino e extensão em outros laboratórios. Podemos também perceber o caráter de extensão deste projeto, já que estes foram doados para as diferentes regiões do Estado do Ceará, na região metropolitana (Pacatuba e Redenção) e interior do Ceará (Acaraú). Além disso, por meio da doação, foi possível uma economia financeira para esses laboratórios, uma vez que esses materiais que estavam estocados puderam ser utilizados, evitando desse modo a compra de novos materiais.

Foi realizada também a destinação correta para alguns resíduos identificados, como por exemplo, soluções ácidas e básicas foram neutralizadas e descartadas na pia. Os resíduos que não puderam ser tratados no próprio laboratório permaneceram estocados aguardando tratamento externo.

\section{CONCLUSÃO}

Os materiais classificados como passivos do Laboratório de Microbiologia Ambiental, estão sendo úteis ao ensino e extensão em outros laboratórios de diferentes regiões do Ceará. Dessa forma a sustentabilidade laboratorial foi alcançada pela reutilização de produtos químicos vencidos.

\section{REFERÊNCIAS}

ANDRADE, S. A.; MASTANTUONO, D.; ABREUB, B. C. G; ICHIKAWAB, V. M.; SANTOS, N. M; FERREIRA, M. S.; BARAZZONED, G. C. Segregação de resíduos químicos por compatibilidade e reatividade no Instituto Butantan. Orbital: The Electronic Journal of Chemistry. v.7, n.1, 2015. < DOI: http://dx.doi.org/10.17807/orbital.v7i1.688

ASSOCIAÇÃO BRASILEIRA DE NORMAS TÉCNICAS. NBR 10004: Classificação dos resíduos sólidos. Rio de Janeiro. 2004.

ASSOCIAÇÃO BRASILEIRA DE NORMAS TÉCNICAS. NBR 14725-4: Produtos químicos - Informações sobre segurança, saúde e meio ambiente Parte 4: Ficha de informações de segurança de produtos químicos (FISPQ). Rio de Janeiro. 2009.

BARROS, A. S. X. Expansão da educação superior no Brasil: limites e possibilidades. Educação e Sociedade, v. 36, n. 131, p. 361-390. 2015. Disponível em: http://www.scielo.br/pdf/es/v36n131/1678-4626-es-36-131-00361.pdf > DOI: http://dx.doi.org/10.1590/ES0101-7330201596208. 
BRASIL. Ministério do Meio Ambiente. Lei $n^{\circ}$ 12.305, de 2 de agosto de 2010, que institui a Política Nacional de Resíduos Sólidos. Disponível em: http://www.mma.gov.br/port/conama/legiabre.cfm?codlegi=636.

FERREIRA, C. P.; GONZALEZ, M. H.; VIANA, L. S. Gestão e gerenciamento de resíduos químicos dos laboratórios didáticos do departamento de química e ciências ambientais e do entreposto de resíduos do IBILCE/UNESP. Fórum Ambiental da Alta Paulista. v.12, n.1, 2016.> DOI: 10.17271/1980082712120161368

JARDIM, F.W. Gerenciamento de resíduos químicos em laboratórios de ensino e pesquisa. Campinas: Química Nova, v. 21 (5), p. 671-673, 1998. Disponível em: http://www.scielo.br/pdf/qn/v21n5/2943.pdfDOI: 10.1590/S0100-

$\underline{40421998000500024}$

KER, A. B.; FREITAS, D. P.; NUNES, F. R. G; CRUZ, S. L.; PINTO, T. G. et. al. Composição gravimétrica dos resíduos sólidos produzidos no campus I do Centro Universitário FAESA. Revista Científica Faesa, v.13, n.1, p. 48-53, 2017. Disponível em: http://revista.faesa.br/revista/index.php/Faesa/article/view/212.

MACHADO, P. F. L.; MÓL, G. S. Resíduos e rejeitos de aulas experimentais: o que fazer?. Química Nova na Escola, v. 29, n. 3. p. 38-41. 2008. Disponível em: http://qnesc.sbq.org.br/online/qnesc29/09-EEQ-4007.pdf.

NASCIMENTO, E. S. L; FILHO, A. T. Chemical waste risk reduction and environmental impact generated by laboratory activities in research and teaching institutions. Brazilian Journal of the Pharmaceutical Sciences, v. 46, n. 2, 2010. Disponível em: http://www.scielo.br/scielo.php? script=sci_arttext\&pid=S198482502010000200004.

http://dx.doi.org/10.1590/S1984-82502010000200004.

NOLASCO, F. R.; TAVARES, G. A.; BENDASSOLLI, J. A. Implantação de programas de gerenciamento de resíduos químicos laboratoriais em universidades: análise crítica e recomendações. Engenharia Sanitária Ambiental, v.11, n.2, p. 118-124, 2006. Disponivel em: http://www.scielo.br/scielo.php? script=sciarttex\&pid=S1413-41522006000200004> http://dx.doi.org/10.1590/S1413-522006000200004

SILVA, J. M.; RODRIGUES, A. B.; SAMPAIO, F. S. O.; OLIVEIRA, E. M. S.; TORRES, S. S. S. et al.. Gerenciamento de Resíduos Laboratoriais: A Experiência do PROGERE- UFC. Extensão em Ação, v.1, n.8, 2015. Disponível em: $<$ http://www.revistaprex.ufc.br/index.php/EXTA/article/view/203>. 